\title{
Prevalence and CT-Scan Presentations of Brain Malformations in Children at a University-Affiliated Mother and Child Hospital (Cameroon)
}

\author{
Boniface Moifo, ${ }^{1,2}$, Rosine Azegha Jiotsa ${ }^{1}$, Seraphin Nguefack ${ }^{1,3}$, Sandra Tatah ${ }^{1}$, Evelyne Mah ${ }^{1,3}$, \\ Félicité Dongmo Nguefack ${ }^{1,3}$, Elie Mbonda ${ }^{1,3}$ \\ ${ }^{1}$ Faculty of Medicine and Biomedical Sciences, The University of Yaounde 1, Yaounde, Cameroon \\ ${ }^{2}$ Radiology Department, Yaounde Gynaeco-Obstetric and Pediatric Hospital, Yaounde, Cameroon \\ ${ }^{3}$ Department of Pediatrics and Pediatric Sub-Specialties, Yaounde Gynaeco-Obstetrics and Pediatric Hospital, Yaounde, Cameroon \\ Email: ^bmoifo@yahoo.fr
}

How to cite this paper: Moifo, B., Azegha Jiotsa, R., Nguefack, S., Tatah, S., Mah, E., Dongmo Nguefack, F. and Mbonda, E. (2017) Prevalence and CT-Scan Presentations of Brain Malformations in Children at a University-Affiliated Mother and Child Hospital (Cameroon). Open Journal of Medical Imaging, 7, 220-228.

https://doi.org/10.4236/ojmi.2017.74020

Received: September 24, 2017

Accepted: November 17, 2017

Published: November 20, 2017

Copyright $\odot 2017$ by authors and Scientific Research Publishing Inc. This work is licensed under the Creative Commons Attribution International License (CC BY 4.0).

http://creativecommons.org/licenses/by/4.0/

\begin{abstract}
Background: Brain malformations (BMs) are congenital abnormalities of the shape or structure of the brain, with corpus callosum agenesis known as the most frequent. Diagnosis of most BMs can be prenatal with ultrasound and fetal magnetic resonance imaging (MRI); post-natal diagnosis is based on transfontanellar ultrasound, CT-scan, and head MRI which is the imaging gold standard technique. MRI has been recently introduced and the CT-scan was previously the reference technique for the diagnosis of BMs in our context. Almost no publication has been made in sub-Saharan Africa on the clinical and CT scan characteristics of Brain malformations in children. Objective: The aim of this study was, in the absence of MRI, to describe the clinical and CT-scan presentations of brain malformations in children at the Yaounde Gynaeco-Obstetric and Pediatric Hospital (YGOPH). Patients and method: This was a cross-sectional descriptive study conducted from February to May 2016 at the YGOPH, including all children of 15-year-old and less with BM diagnosed on CT-scan at YGOPH between April 2006 and March 2016. The studied variables were clinical (age at diagnosis, sex, clinical manifestations) and CT findings (type of cerebral malformation). The data was analyzed using the SPSS 20.0 software with the estimation of hospital prevalence of BMs, frequencies and associations. The chi-square test was used to seek for an association between variables. The threshold of significance was $p<0.05$. Results: The prevalence of BMs was $0.52 \%$, with 29 cases of BMs identified out of 5590 patients followed up at the pediatric neurology outpatient unit over a period
\end{abstract}


of 10 years. The mean age at diagnosis was 37.2 months ( 3.1 years) and the most represented age groups were 0 - 1 year (37.9\%) and 1 - 5 years (37.9\%). The sex ratio was 0.81 ( $55.2 \%$ girls). The clinical presentation was represented by neurological signs (93.1\%) with convulsions (65.5\%) and psychomotor retardation (58.8\%) associated with skin lesions (34.5\%) and/or facial dysmorphic features (27.6\%). BM was suspected on antenatal ultrasound in $14.3 \%$ of cases (4/28). Abnormalities of cortical development accounted for $65.5 \%$ of BM followed by abnormalities of brain separation (31\%). Tuberous sclerosis complex was the most common BM (31\%) followed by agenesis of the corpus callosum (27.6\%). The presence of dysmorphic facial features was associated $(p=0.007)$ with disorders of brain separation (DBS) while the presence of cutaneous lesions was associated $(p=0.013)$ with anomalies of the cortical development (ACD) especially tuberous sclerosis complex. Conclusion: BMs are infrequent, dominated by tuberous sclerosis complex and agenesis of the corpus callosum. They are mainly revealed by convulsions and psychomotor retardation. Efforts are needed to improve antenatal diagnosis and facilitate access to cerebral MRI.

\section{Keywords}

Cerebral Malformations, Psychomotor Retardation, Convulsions,

Tuberous Sclerosis Complex, Corpus Callosum Agenesis,

Cerebral CT Scan, Sub-Saharan Africa

\section{Introduction}

Brain malformations (BMs) are congenital abnormalities of the shape or structure of the brain. They represent $9 \%$ of isolated malformations and are found in $16 \%$ of polymalformative syndromes [1]. The most frequent $\mathrm{BM}$ is agenesis of the corpus callosum (CCA) [2] [3]. These BMs are due to a halt or a modification of brain development during intrauterine life, regardless of the etiology. BM classification incorporates morphological and genetic criteria [4] [5] [6]. The neural tube closure defects (NTCD), rhombencephalon development anomalies (RDA), disorders of brain separation (DBS), cortical development anomalies (CDA), and growth anomalies (GA) are distinguished [7] [8].

Diagnosis of most BMs can be prenatal with ultrasound and fetal magnetic resonance imaging (MRI) [9] [10]. Post-natal diagnosis is based on transfontanellar ultrasound, CT scan, and head MRI which is the imaging gold standard technique [11].

The clinical manifestations of BM are variable, ranging from refractory epilepsy to neurological and cognitive disorders of various severity, in relation to the stage of onset of disorders and the extent of malformation [12].

In Africa, most studies of BM are performed in North Africa where cortical development anomalies are the most frequent [7]. In Cameroon, BMs are often discovered during investigation for epilepsy or psychomotor retardation [13] 
[14]. MRI has been recently introduced and the CT scan was previously the reference technique for the diagnosis of $\mathrm{BM}$ in our context. To the best of our knowledge, no publication has been made in sub-Saharan Africa on the clinical and CT scan characteristics of BMs in children. The purpose of this study was to describe the clinical and CT-scan aspects of child malformations in the Yaounde Gynaeco-Obstetric and Pediatric Hospital (YGOPH), where there is a specialized pediatric neurology unit.

\section{Patients and Methods}

This was a cross-sectional descriptive study conducted in YGOPH's Radiology and Pediatric Neurology units from February to May 2016.

\subsection{Study Population and Procedure}

It included all children aged 0 - 15 years with brain malformation (BM) confirmed by CT scan between April 2006 and March 2016 at HGOPY.

As a first step, we identified all the medical records of BMs among the files of pediatric neurology outpatients from April 2006 to March 2016. Then we contacted their parents by phone to set an appointment at the outpatient consultation in order to investigate the past history and conduct a physical examination of the child. We then retrieved from the medical records of these children data on presenting complaints, initial clinical examination, CT reports and images, and the evolution since diagnosis. All CT scans were reviewed by a pediatric radiologist and a neuropediatrician of at list ten years' experience. All information was recorded on a pre-conceived data collection sheet.

\subsection{Studied Variables}

The clinical data included: age of child at diagnosis of BM, sex, circumstances of diagnosis, prenatal and postnatal history, psychomotor development, convulsions, skin lesions, facial dysmorphic features and other clinical signs. CT-scan data were grouped into neural tube closure defects (NTCD), rhombencephalon development anomalies (RDA), disorders of brain separation (DBS), cortical development anomalies (CDA), growth anomalies (GA), and associated anomalies. Data on other paraclinical examinations, treatment and progression were also noted.

\subsection{Statistical Analysis}

The collected data was recorded using the CSPro 6 software and analyzed in the SPSS 20.0 software. We used descriptive statistics tools with the calculation of frequencies and associations. The chi-square test was used to find an association between the variables; the significance threshold was $p<0.05$.

\subsection{Ethical Considerations}

The study was approved by the Ethics Committee of the Faculty of Medicine and 
Biomedical Sciences and the Institutional Ethics and Research Committee of the YGOPH. The confidentiality of the information and the informed consent (verbal) of the patients' careers have been assured.

\section{Results}

\subsection{Prevalence of Brain Malformations and Characteristics of the Study Population}

Of the 5590 children in Pediatric Neurology outpatient unit at YGOPH between April 2006 and March 2016, 29 had a brain malformation, giving an in-hospital prevalence of $0.52 \%$. Girls accounted for $55.2 \%$ of the population (16/29), giving a sex ratio of 0.81 . Children's ages at diagnosis varied from 2 months to 156 months (13 years) with an average age at diagnosis of 37.2 months (3.1 years). The age distribution was $37.9 \%$ for children aged less than one year, $37.9 \%$ for those aged 1 to 5 , and $24.2 \%$ for those over 5 years of age. Antenatal suspicion of BM was reported in 4 fetuses (14.3\%) after discovery of hydrocephalus ( 2 cases) and microcephaly (2 cases).

\subsection{Clinical Aspects}

Psychomotor retardation (PMR) was the main reason for consultation leading to the discovery of BM, followed by convulsions (31\%). Concerning clinical examination, 14 patients $(48.3 \%)$ had microcephaly and eight $(27.58 \%)$ had signs of facial dysmorphism (hypotelorism, hypertelorism, low set ears). The cutaneous signs (achromic nevus, shagreen patch) were present in 10 patients that is $34.5 \%$. Twenty-seven patients (93.1\%) had neurological signs (Table 1) dominated by convulsions (65.5\%) and psychomotor retardation (58.8\%).

\subsection{CT Scanning}

Cortical development abnormalities (CDA) were the most common BM group (Table 2) with 19 cases (65.5\%).

The main BMs (Table 2 and Figures 1-3) were tuberous sclerosis complex (TSC) with $31 \%$, followed by agenesis of the corpus callosum (ACC) with $27.6 \%$ and pachygyria (20.7\%). Figure 4 shows the Dandy-Walker malformation.

Table 1. Neurological signs.

\begin{tabular}{ccc}
\hline Neurological signs & Number & Percentage (\%) \\
\hline Convulsions & 19 & 65.5 \\
Psychomotor retardation & 17 & 58.8 \\
Spastic tetraparesis & 9 & 31.0 \\
Axial hypotonia & 4 & 13.8 \\
Peripheral hypotonia & 3 & 10.3 \\
School delay & 3 & 10.3 \\
Other & 3 & 10.3 \\
\hline
\end{tabular}

${ }^{\star}$ Other: absence from school (2 cases) walking disorders (one case). 


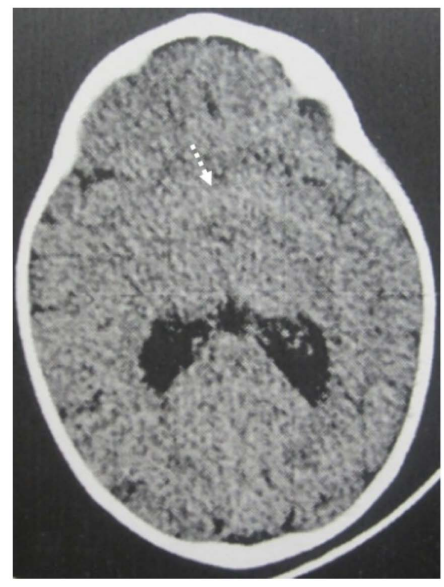

(a)

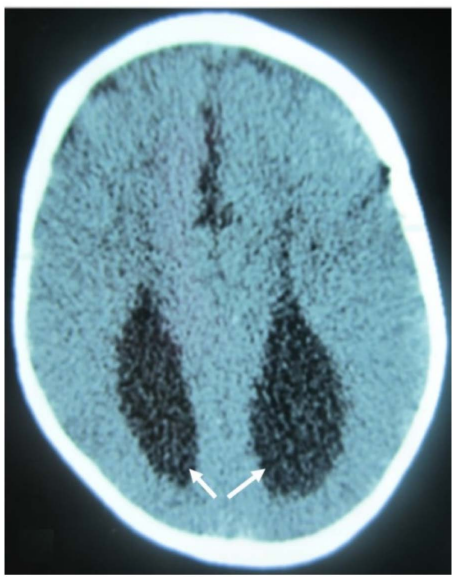

(b)

Figure 1. Cerebral CT scan showing brain separation abnormalities. (a) lobar holoprosencephaly with fusion of the frontal lobes (dot arrow); (b) colpocephaly (white arrows) in corpus callosum agenesis (CCA).

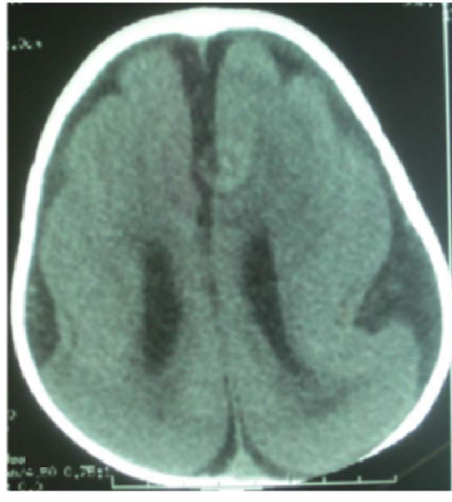

(a)

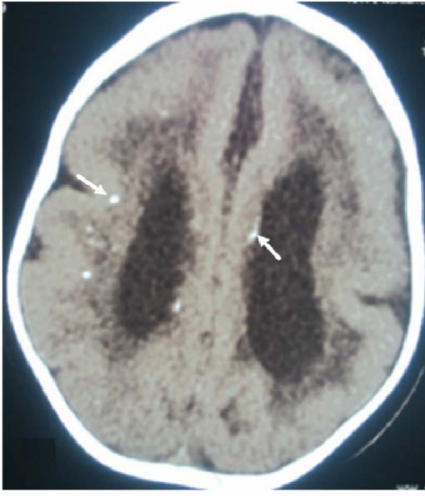

(b)

Figure 2. Cerebral scan; (a) lissencephaly (smooth brain with figure eight appearance); (b) pachygyria (thick cortex) with associated sub-ependymal and parenchymal calcifications (proved CMV infection).

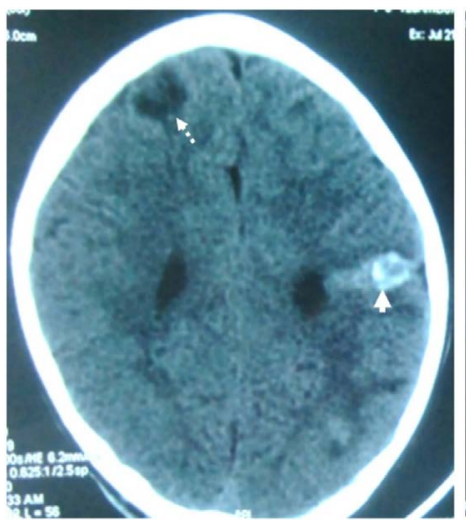

(a)

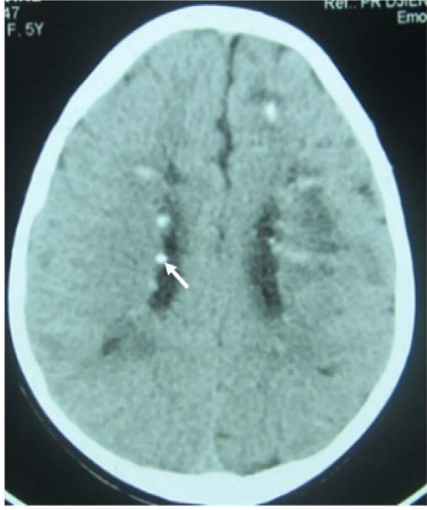

(b)

Figure 3. Cerebral scan showing the lesions characteristic of Tuberous Sclerosis Complex: calcified sub-ependymal nodules (arrow), non-calcified cortical tubers (dot arrow) and calcified cortical tubers (arrow head). 


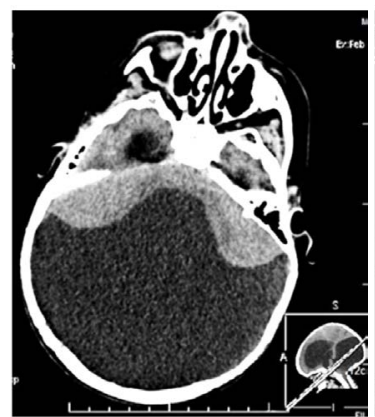

(a)

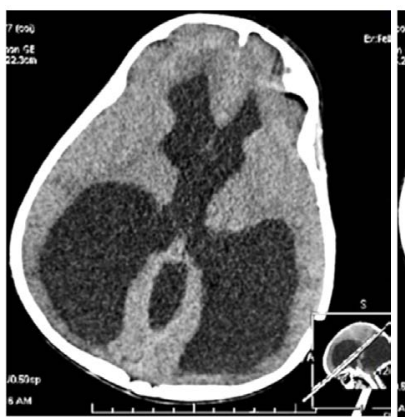

(b)

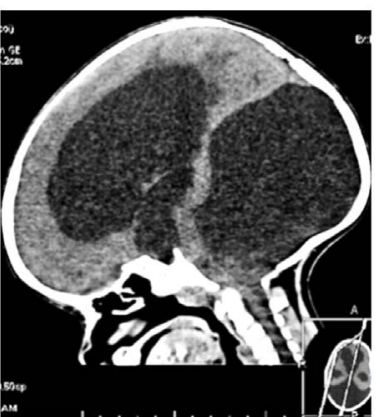

(c)

Figure 4. Cerebral CT scan showing a Dandy-Walker malformation: (a) agenesis of the vermis; (b) triventricular hydrocephalus; (c) cystic expansion of V4, and ascent of the tentorium cerebelli.

Table 2. Distribution of different types of cerebral malformations.

\begin{tabular}{lccc}
\hline Groupe of BM & Type of brain malformations & Number & $\%$ \\
\hline & Tuberous sclerosis complex & 9 & 31 \\
CDA $(\mathrm{n}=19)$ & Pachygyria & 6 & 20.7 \\
& Lissencephaly & 3 & 10.3 \\
& Schizencephaly & 1 & 3.4 \\
DBS $(\mathrm{n}=9)$ & Agenesis of the corpus callosum (ACC) & 8 & 27.6 \\
& Holoprosencephaly & 1 & 3.4 \\
RDA $(\mathrm{n}=3)$ & Agenesis of the vermis & 2 & 6.9 \\
GA $(\mathrm{n}=1)$ & Dandy walker & 1 & 3.4 \\
\hline
\end{tabular}

Cortical Development Anomalies (CDA); Disorders of Brain Separation (DBS); Rhombencephalon Development Anomalies (RDA); Growth Anomalies (GA).

\subsection{Associations}

A statistically significant relationship was found between the group of patients with disorders of brain separation (DBS) and the presence of dysmorphic features $(p=0.007)$. A statistically significant relation was found $(p=0.013)$ between the anomalies of the cortical development (ACD) and the presence of cutaneous lesions, more particularly with tuberous sclerosis complex.

\subsection{Evolution Aspects}

Antiepileptic treatment was instituted in $65.5 \%$ of patients (19/29). However, epileptic seizure control was not yet effective in 13 of 19 patients $(68.4 \%$ of patients on treatment). The mortality rate was $20.7 \%$ that is 6 patients ( 3 cases of lissencephaly, 1 case of TSC, 1 case of ACC and 1 case of holoprosencephaly).

\section{Discussion}

Over 10 years period (2006-2016) we have check off 29 cases of brain malforma- 
tions with a hospital prevalence of $0.52 \%$ in neuropediatric consultations. Our result remains coherent with the frequency of malformations in the nervous system at birth which is $5-10$ per 1000 births [4]. This group of infrequent affections has been the subject of several studies in particular in Morocco and Tunisia [8] [11].

The age of the patients in our study varied from 2 months to 13 years, similar to that found in Tunisia by Hammou et al. in 2002, which was 1 month to 16 years [8]. The mean age at diagnosis of BM in our study was 3.1 years. This result differs from that found by Houmada et al. [11] in 2015 in Morocco with patients of 7 days to 4 years old.

The female sex was predominant in our study. Houmada and Bennouna in Morocco in 2015 [7] [11] found the same observation. On the other hand, Hammou et al. [8] found a male predominance. The sex ratio in BMs varies according to the type of study and BM. Thus, Chateil et al. in France found a male predominance in agyria-pachygyria [15].

The main presenting complaints leading to discovery of BM were psychomotor retardation and convulsions as in the studies of Bennouna et al. [7] and Hammou et al. [8]. Indeed, in these studies the most frequent BMs were cortical development anomalies, and these BMs are known causes of refractory epilepsy [16] [17].

BMs were suspected in antenatal period in $14.3 \%$ of the fetuses with hydrocephalus or microcephaly. This low rate raises questions concerning antenatal screening for BM, which are more often post-natal discoveries following clinical manifestations. It is recommended that at least three obstetric ultrasounds be performed, one in each trimester of pregnancy at a specified time, in order to evaluate, among other things, the development and morphology of CNS structures [18]. And in some cases fetal MRI and genetic analysis are recommended [19] in addition of fetal ultrasound.

Microcephaly was found in $48.3 \%$ of our patients. This microcephaly may result either from decreased neuronal and glial proliferation in germinal areas or from increased apoptosis [20]. Its antenatal discovery must draw our attention and prompt investigation of an embryofetopathy.

Neurological signs dominated the clinical picture in our study. It was essentially convulsions, psychomotor retardation and tone disorders. Convulsions and psychomotor retardation are the most commonly reported clinical signs in the series of brain malformations [8] [11] [20] [21]. These signs were sometimes associated with cutaneous abnormalities and dysmorphic signs. Indeed, facial dysmorphism can be found in certain brain malformations, especially those of the median line [19] [22]. Similarly, BMs such as tuberous sclerosis complex are associated with cutaneous abnormalities such as the appearance of shagreen patches, hypomelanotic spots [23] [24] [25].

Anomalies of cortical development were most frequent same as in Bennouna et al. [7] study. Hammou et al. [8] found a clear predominance of growth anomalies followed by anomalies of cortical development. In addition, TSC was the 
most common BM in our study followed by ACC. This predominance of TSC compared to ACC in our study can be explained by the fact that ACC can remain asymptomatic for a long time and may be accidentally discovered [2] unlike the TSC, where seizures are often forefront features [23] [24].

As limitations: some BMs such as heterotopia or focal dysplasia are difficult to diagnose with CT-scan and can be missed; retrospective review of patient files is source of missing or incomplete data.

\section{Conclusion}

Brain malformations are uncommon in our setting with an in-hospital prevalence of $0.52 \%$. They are dominated by the TSC and ACC. The main clinical manifestations are psychomotor retardation and seizures associated or not with dysmorphic and cutaneous signs. The rate of antenatal suspicion of BM is low.

\section{Conflict of Interest}

The authors state that they have no conflict of interest in relation to this study.

\section{References}

[1] Lépinard, C. (2011) Chapter 11 - Brain Malformations. In: Bonneau, D., Ed., Prenatal Diagnosis in Practice, Elsevier Masson, Paris, 103-130.

[2] Lefevre, J.-P. (2013) Isolated Agenesis of the Corpus Callosum: From Antenatal Diagnosis to Pediatric Prognosis. Dissertation, School of Midwives, Caen.

[3] Bedeschi, M.F., Bonaglia, M.C., Grasso, R., Pellegri, A., Garghentino, R.R., Battaglia, M.A., et al. (2006) Agenesis of the Corpus Callosum: Clinical and Genetic Study in 63 Young Patients. Pediatric Neurology, 34, 186-193. https://doi.org/10.1016/j.pediatrneurol.2005.08.008

[4] Mustard, M.L. and Fallet-Blanco, C. (2004) Fetal Malformative Neurological Pathology. EMC-Pediatrics, 1, 210-231.

[5] Sarnat, H.B. (2000) Molecular Genetic Classification of Central Nervous System Malformations. Journal of Child Neurology, 15, 675-687. https://doi.org/10.1177/088307380001501007

[6] Sarnat, H.B. and Flores-Sarnat, L. (2004) Integrative Classification of Morphology and Molecular Genetics in Central Nervous System Malformations. American Journal of Medical Genetics Part A, 126A, 386-392. https://doi.org/10.1002/ajmg.a.20663

[7] Bennouna, S. (2015) Contribution of MRI in Brain Malformations. Dissertation, Faculty of Medicine and Pharmacy, Fez.

[8] Hammou, C., Douira, W., Abbes, R., Kechaou, I., Bellagha, I. and Hammou, A. (2004) Contribution of MRI in the Exploration of Brain Malformations. Journal de Radiologie, 85, 1558. https://doi.org/10.1016/S0221-0363(04)77866-X

[9] Hornoy, P., Mezzetta, L. and Adamsbaum, C. (2011) Antenatal Detection of Brain Anomalies in Ultrasound. Journal de Radiologie, 92, 111-117. https://doi.org/10.1016/j.jradio.2010.12.003

[10] Garel, C. (2010) Antenatal Brain Imaging and Postnatal Follow-Up. Archives de Pédiatrie, 17, 733-734. https://doi.org/10.1016/S0929-693X(10)70083-X 
[11] Houmada, M.Y., Bennouna, S., Maaroufi, M., Boubbou, M. and Tizniti, S. (2015) Brain Malformations in Children. Revue Neurologique, 171, A129-A130.

[12] Nguefack, S., Gams, D.M., Moifo, B., Mah, E., Chiabi, A., Bogne, J.B., et al. (2014) Clinical and Etiological Aspects of Focal Epilepsy of the Child at the Yaounde Gynaeco-Obstetric and Pediatric Hospital (Cameroon). Le Mali Medical, 29, 4-12.

[13] Nguefack, S., Kamga, K.K., Moifo, B., Chiabi, A., Mah, E. and Mbonda, E. (2013) Causes of Developmental Delay in Children of 5 to 72 Months Old at the Child Neurology Unit of Yaounde Gynaeco-Obstetric and Paediatric Hospital (Cameroon). Open Journal of Pediatrics, 3, 279. https://doi.org/10.4236/ojped.2013.33050

[14] Chateil, J.F., Girault, J.M., Pedespan, J.M., Dousset, V., Castell, J.F. and Diard, F. (1994) Agyries-Pachygyries and Pachygyries of the Child. Contribution of the Imaging. Archives of Pediatrics, 1, 551-560.

[15] Palmini, A. and Holthausen, H. (2013) Focal Malformations of Cortical Development: A Most Relevant Etiology of Epilepsy in Children. Handbook of Clinical Neurology, 111, 549-565.

[16] Spreafico, R. and Tassi, L. (2012) Cortical Malformations. Handbook of Clinical Neurology, 108, 535-557.

[17] Kline-Fath, B.M. and Calvo-Garcia, M.A. (2011) Prenatal Imaging of Congenital Malformations of the Brain. Semin Ultrasound CT MR, 32, 167-188.

https://doi.org/10.1053/j.sult.2011.02.010

[18] Falip, C., Hornoy, P., Millischer Bellaïche, A.E., Merzoug, V. and Adamsbaum, C. (2009) Fetal Magnetic Resonance Imaging (MRI): Indications, Normal and Pathological Aspects. Revue Neurologique, 165, 875-888.

[19] Soto Ares, G., Caron, S. and Jissendi, P. (2007) Malformations of the Brain and Cerebellum. EMC-Radiology and Medical Imaging, 1-24.

[20] Bahi-Buisson, N., Boddaert, N., Straw, Y., Souville, I., Pear Tree, K., Léger, P.-L., et al. (2008) Brain Malformations and Epilepsy: Radioclinical Presentations and Implications for Genetic Diagnosis. Revue Neurologique, 164, 995-1009.

[21] Cottier, J.P., Toutain, A., Hommet, C., Sembely, C., Bosq, M., Texier, N., et al. (2006) Cortical Malformations and Epilepsy: Contribution of MRI. Journal of Radiology, 87, 1621-1634.

[22] Benachi, A. (2010) Anomalies of the Nervous System. In: Practical Conduct in Fetal Medicine, Elsevier Masson, Paris, 35-69.

[23] Moifo, B., Nguefack, S., Neossi Guena, M., Mah, E., Guegang Goudjou, E., Mbonda, E., et al. (2012) Clinical and CT-Scan Presentations in Tuberous Sclerosis Complex: Report of Eight Pediatric Cases Revealed by Epilepsy. Mali Medical, 27, 51-56.

[24] Adamsbaum, C., Merzoug, V. and Kalifa, G. (2008) Brain Imaging of Tuberous Sclerosis of Bourneville in Children. Journal of Neuroradiology, 32, 204-209.

[25] Northrup, H. and Krueger, D.A. (2013) Tuberous Sclerosis Complex Diagnosis Criteria Update: Recommendations of the 2012 Tuberous Sclerosis Complex International Consensus Conference. Pediatric Neurology, 49, 243-254. 